must add the gold of the Altai, and of the silver mines of Nertschinsk, which is 45 pouds; so that the total production is 1825.943 pouds, or about 29,835 kilogrammes $(79,938 \cdot 9$ lbs. troy) of gold in 1847 .

The following table shews the increase of the product of this important working.

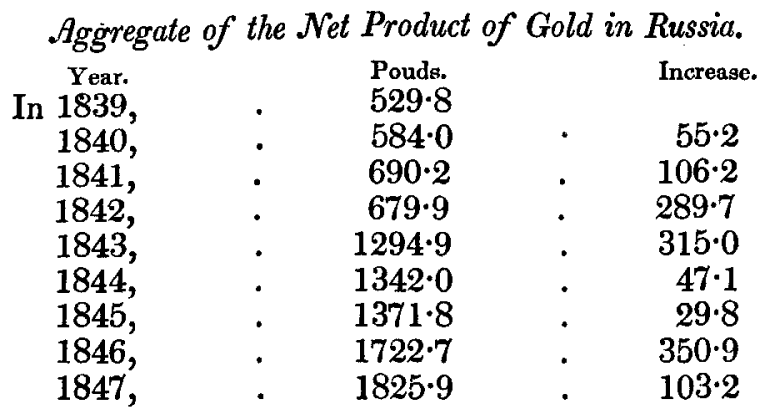

It appears that the space susceptible of being profitably worked-which is left to future generations-is immense.

Translated for the Journal of the Franklin Institute.

Action of Magnetism on all Bodies. By M. Edmond Becquerel.

At the meeting of the 21st May, 1849, of the Academy of Sciences of Paris, M. E. Becquerel communicated a memoir upon the effects of magnetism upon all bodies, the results of which are given in the Comptes Rendus of that date.

The following are the general deductions, the proofs of which will be anxiously looked for by men of science who have paid attention to the recent experiments on diamagnetism.

1. "All bodies becone magnetic, as soft iron itself does, under the influence of a magnet, but in a greater or less degree according to their nature."

2. "The temporary magnetism of a body does not depend upon its mass, but on the manner in which the ether is distributed in the body."

3. "A substance is drawn towards a magnetic centre, by the difference of the actions exerted upon this substance and upon the volume of the medium displaced by it."

The effects were measured by the torsion developed upon small bars of the various substances, by an enormous electro-magnet. The continun? oscillations are prevented by suspending under cach bar a litile jiates ve lead or zinc, immersed in water or a solution of chloride of calcium.

"Measuring in this way the actions exerted upon substances moving in different media, I convinced myself of the enormous influence exerted by the surrounding medium. Thus, common glass, which, in the air, is attracted by the two poles of a magnet, is strongly repelled by these same poles when in solutions of iron or nickel; sulphur and white wax, which, in the air, are repelled by the centres of magnetic action, are, on the con- 
trary, attracted when they are immersed in concentrated solutions of chloride of calcium or chloride of magnesium."

The following are his remarks upon the third general law announced above:-

"Thus a body is attracted or repelled by a magnetic centre, according as it is immersed in a medium less or more magnetic than itself; just as a balloon filled with gas falls to the surface of the earth, or rises in the atmosphere, according as the gas is more or less dense than the air. 'This third principle is, therefore, analogous to the principle of Archinides as to gravity, with this difference: that the latter applies to the mass of the body, while the magnetic intensity developed in a substance, by induction, in nowise depends upon it. Hence it results that the attractions and repulsions exerted upon different bodies, by either pole of a magnet near which they are brought, depend upon the same cause, and not upon two different orders of phenomena. In fact, under the conditions in which the experiments were performed, the attractions and repulsions follow the same laws, and vary in the same way, proportionally to the square of the magnetic intensity."

To explain the fact that all bodies are not attracted by the magnet in vacuo, and that certain substances, such as bismuth, sulphur, phosphorus, \&c., are almost as much repelled in vacuo as in air, "it is necessary to admit that the etherial medium, by means of which magnetic actions are transmitted, is influenced in the same way but in a different degree in a void space, and in one containing matter; and that a void space behaves like a medium more magnetic than the substance which is most repelled: that is to say, bismuth."

"Certain gases, such as nitrogen, nitrous oxide, hydrogen, and carbonic acid, experience no appreciable action from the magnetism, in comparison with the torsion of a silver wire $0.045 \mathrm{~mm} .(0.018 \mathrm{in}$. $)$ in diameter, and 35 centimetres $(14 \mathrm{in}$.) long, but that oxygen is sufficiently magnetic to bave its action easily measured. The air is also magnetic, and as its magnetic power is only about the fifth of that of oxygen, it follows that the effect is due solely to the presence of this latter gas."

"While seeking to shew the magnetic power of oxygen, by some method other than that by the differences of attraction and repulsion, which shew themselves, with small bars of glass and wax immersed successively in vacuo and oxygen, it occurred to me to measure the action exerted by the magnets upon little cylinders of charcoal which condense certain gaseous substances in large proportions. I then found that a small bar of charcoal, which has condensed oxygen, oscillates between the poles of a strong magnet like a small magnetized bar, whilst, in vacuo, it is in general repelled, and always feebly influenced by the action of magnetism."

"Comparing the power of oxygen with that of iron, we conclude that 1 cubic metre $(10.78 \mathrm{cu}$. ft. $)$ of air has an action represented by 11 centigrammes $(1.65$ grains) of iron. If we reflect that the earth is surrounded by a mass of air equal in weight to a stratum of mercury 76 centimetres (30.4 in.) in depth, it may be asked whether such a mass of magnetic gas, continually agitated and submitted to variations, both regular and irregular, of pressure and temperature, does not interfere in the phenomena dependent upon terrestrial magnetism, and perhaps in the diurnal variations 
of the magnetic needle. In fact, if we calculate the magnetic power of this fluid mass, we find it equivalent to an immense sheet of iron, rather more than $\frac{1}{10}$ th of a millimetre $(0.004 \mathrm{in}$.) in thickness, and covering the whole surface of the globe."

"Finally, we may then regard as demonstrated the principles announced above, to wit: that all bodies obey the action of magnetism, but in different degrees, and that the repulsions which shew themselves, of the two poles of a magnet upon certain substances, are due to the fact, that these substances are immersed in a medium more magnetic than themselves, which medium, by its reactions, gives rise to the effects observed. I do not, therefore, admit any difference between what has been called diamagnetism and magnetism properly so called."

For the Journal of the Franklin Institute.

\section{Formula for Making Hyposulphite of Soda.}

A Subscriber in the South, who practises the photogenic process, has met great difficulty in procuring the pure salt called Hyposulphite of Soda, free from sulphate of soda, and will therefore feel greatly obliged if any of your numerous correspondents will publish the formula or receipt for making the same.

In answer to a Subscriber, we offer the following as a good method of preparing hyposulphite of soda.

A strong solution of caustic soda is saturated with sulphur by boiling, and sulphurous acid gaspassed through the clear solution, drawn off from the excess of sulphur, until a small quantity filtered off shows that the liquor has a pale yellow color. It is now filtered, evaporated rapidly until it has a syrupy consistence, again filtered if the liquid be cloudy, mixed well with half its volume of spirit of wine, and set aside to crystalize. The crystals are hyposulphite of soda. When the crystals contain too much sulphur, re-crystalization with alcohol will render them pure. The caustic soda lye is made by dissolving three pounds of crystalized carbonate of soda in two gallons of water, adding one pound of caustic lime previously slacked with one and a half to two quarts of water, boiling the liquid, letting it stand to settle, and decanting the clear liquid. The sulphurous acid is made by heating a thin paste of oil of vitriol and charcoal in a flask, with a tube passing from the cork and under the soda lye. J. C. B.

\section{Machine for Manufacturing Printing Type.}

The Earl of Rosse gave his third Soirée as President of the Royal Society on Saturday last. There were several models and inventions exhibited, the most remarkable amongst the latter being a machine for manufacturing printing types without fusing the metal and pouring it into moulds. The inventor, M. Petit, effects his process by the use of steel dies and matrices, which, by means of powerful pressure, impress the letters, \&c., on copper fashioned into quadrangular strips of indefinite length wound round a cylinder.

The hardness of ordinary copper over type metal is in the proportion of 